\title{
A Priori Bounds in the First Boundary Value Problem in Elasticity
}

\author{
J. H. Bramble ${ }^{1}$ and L. E. Payne ${ }^{1}$
}

(August 22, 1961)

In this paper we obtain bounds for the strain energy, and pointwise bounds for the solution of the first boundary value problem in the equations of elasticity. The inequalities derived are such that the Rayleigh-Ritz technique may be used to improve the bounds.

\section{Introduction}

In a recent paper [2] ${ }^{2}$ the authors gave a method for obtaining bounds in the Dirichlet problem for second-order elliptic partial differential equations. Pointwise bounds were obtained which involved, in addition to the approximating function, only undifferentiated data of the problem. The inequalities were of a priori type, hence only regularity conditions were imposed on the approximating functions.

In this paper we obtain bounds for the strain energy, and pointwise bounds for the solution of the first boundary value problem in the equations of elasticity. Other methods for obtaining bounds, which are based on known variational principles, have been given in the literature (see, for instance, Prager and Synge [9], Diaz and Greenberg [3], Synge [10], and others).

A method based on a priori inequalities was proposed by Payne and Weinberger [8]. Their method involved the introduction of a somewhat complicated unit-tangent vector, and pointwise bounds involved differentiated data. We derive a method which yields pointwise bounds which involve the undifferentiated data of the problem.

In the problem under consideration the displacement vector with components $u_{i}$ satisfies the following system of second-order equations in some $N$-dimensional bounded domain $D$,

$$
L_{i}(u)=u_{i, j j}+\alpha u_{j, j i}=F_{i}
$$

where $\alpha$ involves the elastic constants $\lambda$ and $\mu\left(\alpha=(\lambda+\mu) / \mu=(1-2 \sigma)^{-1}\right.$, $\sigma$ denoting Poisson's ratio), and $F_{i}$ is proportional to the body force. In (1.1), ,ji denotes $\partial^{2} / \partial x_{j} \partial x_{i}$ and a repeated index indicates summation from 1 to $N$, where $N$ denotes the number of dimensions. By specializing to two or three dimensions we can treat the two physically interesting cases. In addition to the differential equation (1.1) the displacement components satisfy the condition

$$
u_{i}=f_{i}
$$

on the boundary $C$ of $D$. Regions which extend to infinity may also be treated if suitable conditions are imposed at infinity.

The strain energy $E(u, u)$ for problem (1.1), (1.2) is defined as:

$$
2 \mu^{-1} E(u, u)=\int_{D}\left[\frac{1}{2}\left(u_{i, j}+u_{j, i}\right)\left(u_{i, j}+u_{j, i}\right)+(\alpha-1)\left(u_{j, j}\right)^{2}\right] d V .
$$

Our problem then is that of obtaining upper and lower bounds for $E(u, u)$ and for the value of $u_{i}(P)$ at a point $P$ in $D$. As we shall see, these bounds follow immediately once we have established certain a priori bounds. Let us suppose that we are able to establish the following inequality for sufficiently smooth functions $w_{i}$ :

$$
E(w, w) \leq K_{1} \oint \eta_{1} w_{i} w_{i} d S+K_{2} \oint_{C} \eta_{2} \operatorname{grad}_{s} w_{i} \cdot \operatorname{grad}_{s} w_{i} d S+K_{3} \int_{D} L_{i}(w) L_{i}(w) d V
$$

\footnotetext{
1 University of Maryland; part-time worker at the National Bureau of Standards.
}

2 Figures in brackets indicate the literature references at the end of this paper. 
where $\eta_{1}$ and $\eta_{2}$ are given positive functions on $C$, and $K_{1}, K_{2}$, and $K_{3}$ are explicitly determined. Here $\operatorname{grad}_{3}$ denotes the tangential projection of the gradient. Now if we set

$$
w_{i}=u_{i}-\phi_{i},
$$

the right hand side of (1.4) will involve only the data of (1.1) and (1.2) and the approximating function $\phi_{i}$. We would then have an a priori bound for $E(u-\phi, u-\phi)$ which could be made small if the functions $\phi_{i}$ could be chosen to approximate the data closely in the mean square sense. But by the triangle inequality

$$
\sqrt{E(\phi, \phi)}-\sqrt{E(u-\phi, u-\phi)} \leq \sqrt{E(u, u)} \leq \sqrt{E(\phi, \phi)}+\sqrt{E(u-\phi, u-\phi)} .
$$

Hence knowledge of the a priori inequality (1.4) would give upper and lower bounds for $E(u, u)$. The inequality (1.4) with $w_{i}=u_{i}-\phi_{i}$ is suitable for application of the Rayleigh-Ritz technique for improving the bounds.

The pointwise bounds will also follow from appropriate a priori inequalities. We concern ourselves then in this paper with the establishment of the necessary a priori inequalities.

\section{Auxiliary In€qualities}

In this section we employ some generalized Green's identities to derive certain inequalities which will be used in sec. 3 . We consider first a function $h$ harmonic in $D$. As indicated in [8, eq 2.4] the function $h$ satisfies the following identity:

$$
\oint_{C}\left[f^{k} n_{k} h_{, i} h_{, i}-2 f^{i} n_{j} h_{, i} h_{, j}\right] d S=\int_{D}\left[f_{, k}^{k} \delta_{i j}-2 f_{. j}^{i}\right] h_{, i} h{ }_{, j} d V
$$

where the $f^{k}$ are components of a piecewise continuous vector field and $\delta_{i j}$ denotes the Kronecker delta. The $n_{j}$ denote the components of the unit normal directed outward from $D$ on $C$. In [8, eq 2.4] we have taken $a^{i j} \equiv \delta_{i j}$ and used the fact that $h$ is harmonic. By breaking up the derivatives of $h$ in the boundary integral into normal and tangential components we obtain

$$
\oint_{C} f^{k} n_{k}\left[\left|\operatorname{grad}_{s} h\right|^{2}-\left(\frac{\partial h}{\partial n}\right)^{2}\right] d S=\int_{D}\left[f_{, k}^{k} \delta_{i j}-2 f_{, j}^{i}\right] h_{, i} h, d V+2 \oint_{C} f^{i} \operatorname{grad}_{s} h \cdot \operatorname{grad}_{s} x_{i} \frac{\partial h}{\partial n} d S
$$

where $\frac{\partial h}{\partial n}$ denotes the normal derivative of $h$ on $C$. If we now choose the $f^{k}$ in such a way that $t=f^{k} n_{k}>0$ on $C$, we obtain the inequality

$$
\oint_{C} t\left(\frac{\partial h}{\partial n}\right)^{2} d S \leq \oint_{C} t\left|\operatorname{grad}_{s} h\right|^{2} d S-2 \oint_{C} f^{i} \operatorname{grad}_{s} h \cdot \operatorname{grad}_{s} x_{i} \frac{\partial h}{\partial n} d S+a D(h, h) .
$$

In (2.3) we have used the notation

$$
D(h, h)=\int_{D} h_{, i} h_{, i} d V
$$

for the Dirichlet integral, and the inequality

$$
\int_{D}\left[2 f_{, j}^{i}-f_{, k}^{k} \delta_{i j}\right] h_{, i} h_{, j} d V \leq a D(h, h) .
$$

The constant $a$ is clearly any upper bound for the largest eigenvalue of the coefficient matrix $2 f_{, j}^{i}-f_{, k}^{k} \delta_{i j}$. Then from Green's first identity, i.e.,

$$
D(h, h)=\oint_{C} h \frac{\partial h}{\partial n} d S
$$


we obtain from $(2.3)$

$$
\oint_{C} t\left(\frac{\partial h}{\partial n}\right)^{2} d S \leq \oint_{C} t\left|\operatorname{grad}_{s} h\right|^{2} d S-\oint_{C}\left[2 f^{i} \operatorname{grad}_{s} h \cdot \operatorname{grad}_{s} x_{i}-a h\right] \frac{\partial h}{\partial n} d S .
$$

Now from Schwarz's inequality we have

$\oint_{C} t\left(\begin{array}{l}\partial h \\ \partial n\end{array}\right)^{2} d S \leq \oint_{C} t\left|\operatorname{grad}_{s} h\right|^{2} d S+\left\{\oint_{C} t^{-1}\left[2 f^{i} \operatorname{grad}_{s} h \cdot \operatorname{grad}_{s} x_{i}-a h\right]^{2} d S \cdot \oint_{C} t\left(\frac{\partial h}{\partial n}\right)^{2} d S\right\}^{1 / 2}$,

from which we obtain, by completing the square,

$$
\begin{aligned}
\left\{\oint_{C} t\left(\frac{\partial h}{\partial n}\right)^{2} d S\right\}^{1 / 2} \leq & \frac{1}{2}\left\{\oint_{C} t^{-1}\left[2 f^{i} \operatorname{grad}_{s} h \cdot \operatorname{grad}_{s} x_{i}-a h\right]^{2} d S\right\}^{1 / 2} \\
& +\left\{\oint_{C} t\left|\operatorname{grad}_{s} h\right|^{2} d S+\frac{1}{4} \oint_{C} t^{-1}\left[2 f^{i} \operatorname{grad}_{s} h \cdot \operatorname{grad}_{s} x_{i}-a h\right]^{2} d S\right\}^{1 / 2},
\end{aligned}
$$

or, using the arithmetic-geometric mean inequality, the cruder inequality

$$
\oint_{C} t\left(\frac{\partial h}{\partial n}\right)^{2} d S \leq \oint_{C} t^{-1}\left[2 f^{i} \operatorname{grad}_{s} h \cdot \operatorname{grad}_{s} x_{i}-a h\right]^{2} d S+2 \oint_{C} t\left|\operatorname{grad}_{s} h\right|^{2} d S .
$$

This inequality bounds the normal derivative of a harmonic function $h$ in terms of $h$ and its tangential derivatives on $C$. (Instead of (2.10) the somewhat better though more involved inequality [8, eq 3.17], with $a^{i j}=\delta_{i j}$, could be used.) In deriving (2.10) we have used the procedures employed in [8]. For completeness and because the case of the harmonic function was not given explicitly in the previous paper we have again presented the derivation of $(2.10)$.

Now let $\psi_{i}$ be the $i$ th component of a vector function which possesses piecewise continuous second derivatives in $D$ and vanishes on $C$. Then consider the following identity:

$$
\begin{aligned}
2 \int_{D} f^{k} \psi_{i, k} L_{i}(\psi) d V=2 \oint_{C} f^{k} \psi_{i, k}\left[\psi_{i, j} n_{j}+\alpha \psi_{j, j} n_{i}\right] d S+\int_{D} f_{, k}^{k}\left[\psi_{i, j} \psi_{i, j}+\alpha \psi_{i, i} \psi_{j, j}\right] d V \\
-2 \int_{D}\left[f_{, j}^{k} \psi_{i, k} \psi_{i, j}+\alpha f_{, i}^{k} \psi_{i, k} \psi_{j, j}\right] d V-\oint_{C} f^{k} n_{k}\left[\psi_{i, j} \psi_{i, j}+\alpha \psi_{i, i} \psi_{j, j}\right] d S .
\end{aligned}
$$

Since $\psi_{i}$ vanishes on $C$ the first and last integrals on the right combine to give (upon rearranging of $(2.11))$

$$
\begin{aligned}
\oint_{C} f^{k} n_{k}\left[\psi_{i, j} \psi_{i, j}+\alpha \psi_{i, i} \psi_{j, j}\right] & d S=2 \int_{D} f^{k} \psi_{i, k} L_{i}(\psi) d V \\
& -\int_{D} f^{k}{ }_{, k}\left[\psi_{i, j} \psi_{i, j}+\alpha \psi_{i, i} \psi_{j, j}\right] d V+2 \int_{D}\left[f_{, j}^{k} \psi_{j, k} \psi_{i, j}+\alpha f_{, i}^{k} \psi_{i, k} \psi_{j, j}\right] d V .
\end{aligned}
$$

Since $f^{i}$ has bounded first derivatives we may easily obtain, for $\alpha$ positive,

$$
2 \int_{D}\left[f_{, j}^{k} \psi_{i, k} \psi_{i, j}+\alpha f_{, i}^{k} \psi_{i, k} \psi_{j, j}\right] d V-\int_{D} f_{, k}^{k}\left[\psi_{i, j} \psi_{i, j}+\alpha \psi_{i, i} \psi_{j, j}\right] d V \leq b \int_{D}\left[\psi_{i, j} \psi_{i, j}+\alpha \psi_{i, i} \psi_{j, j}\right] d V,
$$

where the constant $b$ may be easily determined. (The physically interesting values of $\alpha$ lie in the interval $(1 / 3, \infty)$.)

Note that for $\psi_{i}$ vanishing on $C$ the integral on the right of (2.13) is proportional to the strain energy $E(\psi, \psi)$. Also the quantity inside the square bracket on the left of $(2.12)$ is proportional to the sum of the squares of the suriace tractions. By the arithmetic- 
geometric mean inequality and the fact that $\alpha$ is positive, we have then

$\oint_{C} t\left[\psi_{i, j} \psi_{i, j}+\alpha \psi_{i, i} \psi_{j, j}\right] d S \leq\left[b+\frac{\left[f^{k} f^{k}\right]_{\max }}{\beta}\right] \int_{D}\left[\psi_{i, j} \psi_{i, j}+\alpha \psi_{i, i} \psi_{j, j}\right] d V+\beta \int_{D} L_{i}(\psi) L_{i}(\psi) d V$

for any positive $\beta$. Now since $\psi_{i}$ vanishes on $C$ we have

$$
\int_{D}\left[\psi_{i, j} \psi_{i, j}+\alpha \psi_{i, i} \psi_{j, j}\right] d V=-\int_{D} \psi_{i} L_{i}(\psi) d V
$$

or by Schwarz's inequality

$$
\frac{\int_{D}\left[\psi_{i, j} \psi_{i, j}+\alpha \psi_{i, i} \psi_{j, j}\right] d V}{\int_{D} \psi_{i} \psi_{i} d V} \leq \frac{\int_{D} L_{i}(\psi) L_{i}(\psi) d V}{\int_{D}\left[\psi_{i, j} \psi_{i, j}+\alpha \psi_{i, i} \psi_{j, j}\right] d V} .
$$

However, for $\alpha>0$

$$
\frac{\int_{D}\left[\psi_{i, j} \psi_{i, j}+\alpha \psi_{i, i} \psi_{j, j}\right] d V}{\int_{D} \psi_{i} \psi_{i} d V} \geq \frac{\int_{D} \psi_{i, j} \psi_{i, j} d V}{\int_{D} \psi_{i} \psi_{i} d V} \geq \lambda_{1},
$$

where $\lambda_{1}$ is the first eigenvalue in the fixed membrane problem for $D$. Lower bounds for $\lambda_{1}$ are easily derivable from the Faber-Krahn inequality [5], [6], or from monotony principles. Thus from (2.16) and (2.17) we have

$$
\int_{D} \psi_{i} \psi_{i} d V \leq \frac{1}{\lambda_{1}} \int_{D}\left[\psi_{i, j} \psi_{i, 3}+\alpha \psi_{i, i} \psi_{j, j}\right] d V \leq \frac{1}{\lambda_{1}^{2}} \int_{D} L_{i}(\psi) L_{i}(\psi) d V
$$

The insertion of (2.18) into (2.14) then yields

$$
\oint_{C} t\left[\psi_{i, j} \psi_{i, j}+\alpha \psi_{i, i} \psi_{j, j}\right] d S \leq\left[b / \lambda_{1}+2\left\{\left[f^{k} f^{k}\right]_{\max } / \lambda_{1}\right\}^{\frac{1}{2}}\right] \int_{D} L_{i}(\psi) L_{i}(\psi) d V
$$

where we have made the optimal choice for $\beta$.

\section{A Priori Inequalities}

Let $w_{\imath}$ have piecewise continuous second derivatives in $D$. We seek an a priori bound for $E(w, w)$. To this end we decompose $w_{i}$ as

where

$$
w_{\imath}=\bar{w}_{i}+w_{i}^{\prime}
$$

in $D$ and

$$
L_{i}(\bar{w})=0
$$

on $C$. The function $w_{i}^{\prime}$ then satisfies

$$
\bar{w}_{i}=w_{i}
$$

in $D$ and

$$
L_{i}\left(w^{\prime}\right)=L_{i}(w)
$$

on $C$. Then clearly

$$
w^{\prime}{ }_{i}=0
$$

$$
E(w, w)=E(\bar{w}, \bar{w})+E\left(w_{i}^{\prime} w^{\prime}\right)
$$

since $\bar{w}$ and $w^{\prime}$ are orthogonal in $E$-norm (i.e., $E\left(\bar{w}, w^{\prime}\right)=0$ ). We derive first a bound for 
$E\left(w^{\prime}, w^{\prime}\right)$. From the fact that $w_{i}^{\prime}=0$ on $C$ we have from $(2.18)$, with $\psi_{i}$ replaced by $w_{i}^{\prime}$,

$$
2 \mu^{-1} E\left(w^{\prime}, w^{\prime}\right) \leq \frac{1}{\lambda_{1}} \int_{D} L_{i}\left(w^{\prime}\right) L_{i}\left(w^{\prime}\right) d V=\frac{1}{\lambda_{1}} \int_{D} L_{i}(w) L_{i}(w) d V
$$

Just as one establishes the Dirichlet principle for harmonic functions it is possible in bounding $E(\bar{w}, \bar{w})$ to show that

$$
E(\bar{w}, \bar{w}) \leq E(H, H)
$$

where $H$ is any vector which satisfies the same boundary values as $\bar{w}$ on $C$. Let $H_{i}$ be the components of the harmonic vector which takes on the same boundary values as $\bar{w}_{i}$. Then

$$
\begin{aligned}
E(\bar{w}, \bar{w}) & \leq \bar{\mu} \int_{D} H_{i, j} H_{i, j} d V, \quad \bar{\mu}= \begin{cases}\mu[2+N(\alpha-1)], & \alpha>1 \\
2 \mu & , \alpha \leq 1\end{cases} \\
& =\bar{\mu} \oint_{C} H_{i} \frac{\partial}{\partial n} H_{i} d S,
\end{aligned}
$$

the latter equality following from Green's theorem and the fact that $H_{i}$ is harmonic. Now choose $f^{i}$ in such a way that $t=f^{i} n_{i}>0$ on $C$. Then by the arithmetic-geometric mean inequality we have

$$
E(\bar{w}, \bar{w}) \leq \frac{\bar{\mu}}{\gamma} \oint_{C} t^{-1} H_{i} H_{i} d S+\mu \gamma \oint_{C} t \frac{\partial H_{i}}{\partial n} \frac{\partial H_{i}}{\partial n} d S
$$

for any positive $\gamma$. Now since the $H_{i}$ are harmonic we may use inequality (2.10) for vectors to obtain

$$
\begin{array}{r}
\oint_{C} t \frac{\partial H_{i}}{\partial n} \frac{\partial H_{i}}{\partial n} d S \leq \oint_{C} t^{-1}\left[2 f^{j} \operatorname{grad}_{s} H_{i} \cdot \operatorname{grad}_{s} x_{j}-a H_{i}\right]\left[2 f^{k} \operatorname{grad}_{s} H_{i} \cdot \operatorname{grad}_{s} x_{k}-a H_{i}\right] d S \\
+2 \oint_{C} t \operatorname{grad}_{s} H_{i} \cdot \operatorname{grad}_{s} H_{i} d S .
\end{array}
$$

Now since $H_{i}=\bar{w}_{i}=w_{i}$ on $C$ we have upon combining (3.6), (3.7), (3.10), and (3.11) the desired bound

$$
\begin{aligned}
& 2 \bar{\mu}^{-1} E(w, w) \leq \frac{1}{\gamma} \oint_{C} t^{-1} w_{i} w_{i} d S+\gamma\left\{2 \oint_{C} t \operatorname{grad}_{s} w_{i} \cdot \operatorname{grad}_{s} w_{i} d S\right. \\
& \left.\quad+\oint_{C} t^{-1}\left[2 f^{j} \operatorname{grad}_{s} w_{i} \cdot \operatorname{grad}_{s} x_{j}-a w_{i}\right]\left[2 f^{k} \operatorname{grad}_{s} w_{i} \cdot \operatorname{grad}_{s} x_{k}-a w_{i}\right] d S\right\}+\frac{\mu / \bar{\mu}}{\lambda_{1}} \int_{D} L_{i}(w) L_{i}(w) d V
\end{aligned}
$$

which can clearly be put in the form (1.4) if so desired.

We now derive a bound for the integral of the sum of the squares of the displacement components $w_{i}$ over $D$. This inequality will be used in sec. 5 to derive pointwise bounds for $w_{i}$. We seek then a bound for $\int_{D} w_{i} w_{i} d V$.

To this end we introduce the auxiliary vector $\chi$ with components $\chi_{i}$ defined by

in $D$ and

$$
L_{i}(\chi)=w_{i}
$$

on $C$. Then

$$
\chi_{i}=0
$$

$$
\int_{D} w_{i} w_{i} d V=\int_{D} w_{i} L_{i}(\chi) d V=\oint_{C} w_{i}\left[\chi_{i, j} n_{j}+\alpha \chi_{i, j} n_{i}\right] d S+\int_{D} \chi_{i} L_{i}(w) d V
$$


By Schwarz's inequality for vectors we have

$$
\begin{aligned}
\int_{D} w_{i} w_{i} d V=\int_{D} L_{i}(\chi) L_{i}(\chi) d V \leq\left\{\oint _ { C } t ^ { - 1 } [ w _ { i } w _ { i } + ( w _ { i } n _ { i } ) ^ { 2 } ] d S \oint _ { C } t \left[\chi_{i, i} \chi_{i, 3}\right.\right. \\
\left.\left.+\alpha \chi_{i, i} \chi_{i, i}\right] d S\right\}^{\frac{1}{2}}+\left\{\int_{D} \chi_{i} \chi_{i} d V \int_{D} L_{i}(w) L_{i}(w) d V\right\}^{\frac{1}{2}} .
\end{aligned}
$$

Since $\chi_{i}$ vanishes on $C$ we may use (2.18) and (2.19) with $\psi_{i}$ replaced by $\chi_{i}$ to obtain

$$
\begin{aligned}
{\left[\int_{D} w_{i} w_{i} d V\right]^{\frac{1}{2}} \leq\left\{[ b / \lambda _ { 1 } + 2 ( [ f ^ { k } f ^ { k } ] _ { \operatorname { m a x } } / \lambda _ { 1 } ) ^ { \frac { 1 } { 2 } } ] \oint _ { C } t ^ { - 1 } \left[w_{i} w_{i}\right.\right.} \\
\left.\left.+\left(w_{i} n_{i}\right)^{2}\right] d S\right\}^{\frac{1}{2}}+\left\{\frac{1}{\lambda_{1}^{2}} \int_{D} L_{i}(w) L_{i}(w) d V\right\}^{\frac{1}{2}} .
\end{aligned}
$$

If we should now set $w_{i}=u_{i}-\phi_{i}$ where $u_{i}$ is the solution of (1.1), (1.2) and $\phi_{i}$ is an approximating function then the right hand side of (3.17) would involve $u_{i}$ only through its data. We employ this inequality in the next section for obtaining pointwise bounds for $w_{i}$.

\section{Pointwise Bounds}

In order to obtain pointwise bounds we again decompose $w_{i}$ as in eq (3.1). Pointwise bounds for $\bar{w}_{i}$ are then derived by making use of a mean-value theorem for the solution of the homogeneous equations of elasticity. Such a mean-value theorem was given in the threedimensional case by Aquaro [1] and Synge [10]. The analogous $N$-dimensional result was obtained by Diaz and Payne [4].

Let $S(r)$ be a sphere of radius $r$ and surface $\sum(r)$ with center at the point at which pointwise bounds are sought. We choose the origin of a spherical-coordinate system at this point, and require that $S(r)$ be contained in $D$.

In what follows whenever a Greek index is repeated the summation convention is not to be used, i.e., $u_{\alpha, i} u_{\alpha, i}$ will denote the square of the gradient of a single-displacement component $u_{\alpha}$.

From the mean-value theorem [4, eq 3.9]

$$
r^{N+1} \bar{w}_{\beta}(0)=\frac{N}{2(N+\alpha) w_{N}} \oint_{\Sigma(r)}\left[(2-\alpha) \delta_{\beta j} r^{2}+(N+2) \alpha x_{\beta} x_{j}\right] \bar{w}_{j} d S .
$$

If we now integrate (4.2) with respect to $r$ from the origin to $R$, with $R$ so chosen that $S(R)$ is still contained in $D$, we obtain

$$
\bar{w}_{\beta}(0)=\frac{N(N+2)}{2(N+\alpha) R^{N+2} w_{N}} \int_{S(R)}\left[(2-\alpha) \delta_{\beta j} r^{2}+(N+2) \alpha x_{\beta} x_{j}\right] \bar{w}_{j} d V .
$$

An application of Schwarz's inequality then yields

$$
\begin{aligned}
\left|\bar{w}_{\beta}(0)\right|^{2} & \leq \frac{N}{N+4}\left[\frac{(N+2)}{2(N+\alpha)}\right]^{2}\left\{\frac{N(2-\alpha)^{2}+2 \alpha(2-\alpha)(N+2)+(N+2)^{2} \alpha^{2}}{w_{N} R^{N}}\right\} \int_{D} \bar{w}_{j} \bar{w}_{j} d V \\
& \equiv A(N) R^{-N} \int_{D} \bar{w}_{j} \bar{w}_{j} d V .
\end{aligned}
$$

By our construction we see that $R$ is actually the distance from the origin to the boundary. 
Now from (3.17), since $L_{i}(-) \equiv 0$ it follows that

where

$$
\begin{aligned}
\left|\bar{w}_{\beta}(0)\right|^{2} & \leq A(N) K_{1}^{2} R^{-N} \oint_{C} t^{-1}\left[\bar{w}_{i} \bar{w}_{i}+\left(\bar{w}_{i} n_{i}\right)^{2}\right] d S \\
& =A(N) K_{1}^{2} R^{-N} \oint_{C} t^{-1}\left[w_{i} w_{i}+\left(w_{i} n_{i}\right)^{2}\right] d S
\end{aligned}
$$

$$
K_{1}=\left[b / \lambda_{1}+2\left(\left[f^{k} f^{k}\right]_{\max } / \lambda_{1}\right)^{1 / 2}\right] .
$$

In order to obtain bounds for $w_{\beta}^{\prime}(0)$ we introduce the Kelvin fundamental solution $z_{\beta}^{k}(O, Q)$ which denotes the components of displacement in the $k$ th direction corresponding to a point load at the origin in the $\beta$ th direction, i.e.,

$$
z_{\beta}^{k}= \begin{cases}\frac{1}{4 \pi \mu(\alpha+1)}\left[(\alpha+2) \log (1 / r) \delta_{\beta k}+\alpha x_{\beta} x_{k} r^{-2}\right], & N=2 \\ \frac{1}{2 \mu(N-2)(\alpha+1) w_{N}}\left[(\alpha+2) r^{-(N-2)} \delta_{\beta_{k}}+(N-2) \alpha x_{\beta} x_{k} r^{-N}\right], & N>2 .\end{cases}
$$

For $N=2,3$ these expressions are given in Love [7]. By the analogue of Green's third identity for the equations of elasticity we have

$$
w_{\beta}^{\prime}(0)=\oint_{C} z_{\beta}^{k}\left[w_{k, j}^{\prime} n_{j}+\alpha w_{j, j}^{\prime} n_{k}\right] d S+\int_{D} z_{\beta}^{k} L_{k}\left(w^{\prime}\right) d V .
$$

Since $L_{k}\left(w^{\prime}\right) \equiv L_{k}(w)$ in $D$, for the boundary-value problem under consideration $L_{k}\left(w^{\prime}\right)$ will be known. Hence by Schwarz's inequality

$$
\left|w_{\beta}^{\prime}(0)\right|^{2} \leq\left\{\left[\oint_{C} t^{-1}\left(z_{\beta}^{k} z_{\beta}^{k}+\left[z_{\beta}^{k} n_{k}\right]^{2}\right) d S \oint_{C} t\left(w_{i, j}^{\prime} w_{i, j}^{\prime}+\alpha w_{i, i}^{\prime} w_{j, j}^{\prime}\right) d S\right]^{1 / 2}+\left|\int_{D} z_{\beta}^{k} L_{k}(w) d V\right|\right\}^{2} .
$$

Now since $w_{i}^{\prime}$ vanishes on $C$ we may use inequality (2.19) to bound the second boundary integral on the right of (4.8); i.e.,

$$
\left|w_{\beta}^{\prime \prime}(0)\right|^{2} \leq\left\{\left[K_{1} \oint_{C} t^{-1}\left(z_{\beta}^{k} z_{\beta}^{k}+\left[z_{\beta}^{k} n_{k}\right]^{2}\right) d S \int_{D} L_{j}(w) L_{\jmath}(w) d V\right]^{1 / 2}+\left|\int_{D} z_{\beta}^{k} L_{k}(w) d V\right|\right\}^{2} .
$$

Now since

$$
\left|w_{\beta}(0)\right|^{2} \leq 2\left\{\left|\bar{w}_{\beta}(0)\right|^{2}+\left|w_{\beta}^{\prime}(0)\right|^{2}\right\}
$$

we have by inequalities (4.4) and (4.10) a bound for any one of the displacement components at the origin.

We may also obtain pointwise bounds for the derivatives of $u_{\beta}$ at points in $D$. Again we first obtain an a priori bound for the derivative of a general function $w_{\beta}$ and interpret our results for $w_{\beta}=u_{\beta}-\phi_{\beta}$. As before $w_{\beta}$ is decomposed as $w_{\beta}=\bar{w}_{\beta}+w_{\beta}^{\prime}$. The pointwise bounds for the derivatives of $\bar{w}_{\beta}$ are again obtained by use of mean-value inequalities while the fundamental solution (4.6) is used to obtain derivatives of $w_{\beta}^{\prime}$. Diaz and Payne [4, eq 4.14] have shown that the stress component $\bar{\tau}_{\beta \gamma}$ defined as

$$
\bar{\tau}_{\beta \gamma}=\mu\left[\left(\bar{w}_{\beta, \gamma}+\bar{w}_{\gamma, \beta}\right)+(\alpha-1) \bar{w}_{k, k} \delta_{\beta \gamma}\right]
$$

satisfies the mean value theorem

$$
\begin{array}{r}
r^{N+3} \bar{\tau}_{\beta \gamma}(0)=\frac{\mu N}{w_{N}(N+2 \alpha+2)}\left[(N+2)(1-\alpha) \oint_{\Sigma(r)} r^{2}\left(\bar{w}_{\beta} x_{\gamma}+\bar{w}_{\gamma} x_{\beta}\right) d S+\alpha(N+2)(N+4) \oint_{\Sigma(r)} x_{\beta} x_{\gamma} x_{k} \bar{w}_{k} d S\right. \\
\left.+\left(2 \alpha^{2}-4 \alpha-N-2\right) \delta_{\beta \gamma} \oint_{\Sigma(r)} r^{2} \bar{w}_{k} x_{k} d S\right] .
\end{array}
$$


Upon integrating with respect to $r$ as before from 0 to $R$ we obtain

$$
\begin{aligned}
R^{N+4} \bar{\tau}_{\beta \gamma}(0)=\frac{\mu N(N+4)}{w_{N}(N+2 \alpha+2)} \int_{S(R)}\left[(N+2)(1-\alpha) r^{2}\left(x_{\beta} \delta_{\gamma k}+x_{\gamma} \delta_{\beta R}\right)\right. & \left.+\alpha(N+2)(N+4) x_{\beta} x_{\gamma} x_{k}+\left(2 \alpha^{2}-4 \alpha-N-2\right) \delta_{\beta \gamma} x_{k}\right] \bar{w}_{k} d V,
\end{aligned}
$$

where $S(R)$ again is required to lie interior to $D$. An application of Schwarz's inequality then yields

$$
\left|\bar{\tau}_{\beta \gamma}(0)\right|^{2} \leq K_{\beta \gamma}(N) R^{-(N+2)} \int_{S(R)} \bar{w}_{k} \bar{w}_{k} d V,
$$

where $K_{\beta \gamma}(N)$ is an easily computable function of $N$.

To obtain a bound for $\tau_{\beta \gamma}^{\prime}(0)$ we differentiate (4.7) to obtain

$$
\tau_{\beta \gamma}^{\prime}(0)=\oint_{C} \tau_{\beta \gamma}\left(z^{k}\right)\left[w_{k j}^{\prime} n_{j}+\alpha w_{j, j}^{\prime} n_{k}\right] d S+\int_{D} \tau_{\beta \gamma}\left(z^{k}\right) L_{k}\left(w^{\prime}\right) d V
$$

where we have used the notation

$$
\mu^{-1} \tau_{\beta \gamma}\left(z^{k}\right)=\frac{\partial z_{\beta}^{k}(P, Q)}{\partial x_{\gamma}}+\frac{\partial z_{\gamma}^{k}(P, Q)}{\partial x_{\beta}}+\alpha \frac{\partial z_{l}^{k}(P, Q)}{\partial x_{l}} \delta_{\beta \gamma}
$$

the differentiation being taken with respect to the variables of the point $P$. Thus the $\tau_{\beta \gamma}\left(z^{k}\right)$ occurring in $(4.1 \dot{5})$ is formed by first taking $\approx_{i}^{k}(P, Q)$ (the displacement at $Q$ due to a point load at $P$ ) performing the indicated differentiation with respect to the variables of the point $P$ and then evaluating at the origin. As before, from Schwarz's inequality we obtain

$$
\begin{aligned}
\left|\tau_{\beta \gamma}^{\prime}(0)\right|^{2} \leq\left(\left\{\oint_{C} t^{-1}\left[\tau_{\beta \gamma}\left(z^{k}\right) \tau_{\beta \gamma}\left(z^{k}\right)+\left(\tau_{\beta \gamma}\left(z^{k}\right) n_{k}\right)^{2}\right] d S\right.\right. & \\
& \left.\left.\oint_{C} t\left(w_{k, l}^{\prime} w_{k, l}^{\prime}+\alpha w_{k, k}^{\prime} w_{l, l}^{\prime}\right) d S\right\}^{\frac{1}{2}}+\left|\int_{D} \tau_{\beta \gamma}\left(z^{k}\right) L_{k}(w) d V\right|\right)^{2} .
\end{aligned}
$$

The insertion of (2.19) into (4.17) then leads to the result

$$
\left|\tau_{\beta \gamma}^{\prime}(0)\right|^{2} \leq\left\{M_{\beta \gamma}(N)\left[\int_{D} L_{j}(w) L_{j}(w) d V\right]^{\frac{1}{2}}+\left|\int_{D} \tau_{\beta \gamma}\left(z^{k}\right) L_{k}(w) d V\right|\right\}^{2}
$$

where $M_{\beta \gamma}(N)$ is an easily computed constant. The desired bound for $\tau_{\beta \gamma}(0)$ now follows since

$$
\left|\tau_{\beta \gamma}(0)\right|^{2} \leq 2\left\{\left|\bar{\tau}_{\beta \gamma}(0)\right|^{2}+\left|\tau_{\beta \gamma}^{\prime}(0)\right|^{2}\right\} \text {. }
$$

\section{References}

[1] Aquaro, G., Un teorema di media per le equazioni dell'elasticità, Riv. Mat. Univ. Parma 1, 419-424 (1950).

[2] Bramble, J. H. and Payne, L. E., Bounds for solutions of second order elliptic partial differential equations. Contributions to Differential Equations (in press).

[3] Diaz, J. B. and Greenberg, H. J., Upper and lower bounds for the solution of the first boundary value problem of elasticity. Quart. Appl. Math. 6, 326-331 (1948).

[4] Diaz, J. B. and Payne, L. E., Mean value theorems in the theory of elasticity. Proc. Third U.S. Congress Appl. Mech., Brown Univ., pp. 293-303 (1958).

「5] Faber, G., Beweis, dass unter aller homogenen Membranen von gleicher Fläche und gleicher Spannung die Kreisförmige den tiefsten Grundton gibt, Sitzber. bayer. Akad. Wiss., pp. 169-172 (1923).

[6] Krahn, E., Über eine von Rayleigh formulierte Minimaleigenschaft des Kreises, Math. Ann. 94, 97-100 (1924); see also, U̇ber Minimaleigenschaften der Kugel in drei und mehr Dimensionen, Acta. Comm. Univ. Dorp. A 9, 1-44 (1926).

[7] Love, A. E. H., A treatise on the mathematical theory of elasticity (Dover, New York, 1944).

[8] Payne, L. E. and Weinberger, H. F., New bounds for solutions of second order partial differentialequations, Pac. J. Math. 8, 551-573 (1958).

[9] Prager, W. and Synge, J. L., Approximations in elasticity based on the concept of function space, Quart. Appl. Math. 5, 241-269 (1947).

[10] Synge, J. L., Upper and lower bounds for the solutions of problems in elasticity, Proc. Roy. Irish Acad. 53, 41-64 (1950).

(Paper 65B4-66) 


\title{
Publications of the National Bureau of Standards*
}

\author{
(Including Papers in Outside Journals)
}

\section{Selected Abstracts}

The nonsingular embedding of transition processes with a more general framework of coupled variables, J. Heading, $J$. Research NBS 65D (Radio Prop.), No. 6, (Nov.-Dec. 1961). 75 cents.

Reflection and coupling processes exhibited by plane electromagnetic waves propagated in an inhomogeneous horizontally-stratified anisotropic ionosphere are associated with discrete transition points or with continuous coupling regions. These arise when the fourth order differential equations are written in first order coupled form, and many terms in these equations become infinite at the transition points. This procedure is rendered more precise by means of a special linear transformation that reformulates the equations in a new way, thereby exhibiting the manner in which local coupling processes are embedded in the more general background process of otherwise independently propagated characteristic waves. To exhibit the power of the matrix algebra involved, the case of an arbitrary number of characteristic waves is considered; moreover, Försterling-type coupled equations are produced in a more generalized form than hitherto considered, and a discussion of the equations governing continuous coupling completes the paper.

The robustness of life testing procedures derived from the exponential distribution, M. Zelen and M. C. Dannemiller, Technometrics 3, No. 1, 29-49 (Feb. 1961).

Almost all the statistical procedures in current use for evaluating the reliability of components or equipment rest on the assumption that the failure times follow the exponential distribution. However, in practical situations one rarely has enough data to determine whether failure times are actually exponential. This paper studies the behavior of several statistical life testing procedures based on the exponential failure law if the true failure law is the Weibull distribution. It is found that these statistical techniques, which are widely used, are very sensitive to departures from initial assumptions. Applying these techniques to life test data when the exponential failure law is not satisfied may result in substantially increasing the probability of accepting components or equipments having poor mean-time-to-failure.

This paper also develops convenient analytic techniques for approximating (i) the distribution of sums of independent random variables, and (ii) the characteristics of sequential procedures, for non-negative random variables. These techniques are based on a general life distribution which consists of an expansion of generalized Laguerre polynomials. Application of these methods to the Weibull distribution yields excellent results.

Applications of graphs and Boolean matrices to computer programming, R. B. Marimont, SI AM Rev. 2, No. 4, 259-268 (Oct. 1960).

It is shown how the techniques of graph theory and Boolean matrices may be used in the analysis of digital computer programs for the elimination of inconsistencies and redundancies. The inadequacy of the present methods to deal with the more complicated problems of computer networks is illustrated by discussion of some important unsolved problems.

On some partıal differential ecuations of Brownian motion of a free particle, A. Ghaffari, (Abstract) Proc. Intern. Conf. on Partial Differential Equations and Continuum Mechanics (Univ. of Wisconsin, Madison, Wisc., June $7-15,1960)$, 348-350 (1961).

The most general one-dimensional solutions of the ChapmanKolmogoroff functional equation are well known as series of parabolic cylinder functions. The present paper gives a derivation of the corresponding parabolic partial differential equations and their simplification and solution for a special case. These solutions differ from those of A. N. Kolmogoroff and are of class $L(2)$ over $(-\infty,+\infty)$. The same method furnishes the most general one-dimensional solutions, as series of Laguerre polynomials which converge uniformly over $(0,+\infty)$ under certain conditions.

Nonequilibrium processes in isotopically disordered crystals. Dependence on degree of order, R. Zwanzig, J. Math. Phys., 2, No. 3, 370-372 (Mar.-June 1961)

A previous paper [A. A. Maradudin, G. H. Weiss, and D. H. Jepsen, J. Math. Phys. 2, 349 (1961)] has shown that approximate normal modes of isotopically disordered crystals decay irreversibly when the arrangement of isotopes is completely random. These results are generalized to crystals with an arbitrary degree of order. In particular, it is shown irreversible behavior occurs whenever the spatial correlation between isotopic species extends over a fixed finite range, as the size of the crystal tends to infinity.

A diffraction theory for LF sky-wave propagation, J. R. Wait, J. Geophys. Research 66, No. 6, 1713-1724 (June 1961), An additional note, J. R. Wait and A. M. Conda, J. Geophys. Research 66, No. 6, 1725-1729 (June 1961).

The concept that radio waves propagate from transmitter to receiver via discrete ionospheric reflections (i.e., hops) is put on a sound theoretical basis. The earth and the ionosphere are represented by two (smooth) concentric spherical surfaces. The (local) reflecting characteristics of these boundaries are assumed to be known. It is shown that the $m$ 'th hop sky wave may be generally represented by a complex integral. The geometrical-optical representations for the field are retrieved as a special case. For low-radio-frequencies, it is shown that these optical-type formulae, which are often used, have severe limitations. Generally, they are not valid near the caustic points. Techniques for evaluating the complex integral for the sky waves near and beyond the caustics are then discussed in some detail. Where possible, a physical interpretation of the results is given.

\section{List of Titles}

Journal of Research 65A (Phys. and Chem.), No. 6 (Nov.Dec. 1961) 75 cents.

Comparison of lens response for sinusoidal and square-wave targets at several focal positions. S. H. Emara.

Wavelength shifts in $\mathrm{Hg}^{198}$ as a function of temperature. S. H: Emara.

Variability of spectral tristimulus values. I. Nimeroff, J. R. Rosenblatt, and M. C. Dannemiller.

Extension of the Flory-Rehner theory of swelling to an anisotropic polymer system. S. D. Bruck.

Fiber structure-property relationships: a disulfide-crosslinked self-crimping polyamide. S. D. Bruck.

Acidity functions. Values of the quantity $p\left(a_{\mathrm{H}} \gamma_{\mathrm{Cl}}\right)$ for buffer solutions from 0 to $95{ }^{\circ} \mathrm{C}$. R. G. Bates and R. Gary.

$2,3-O-I s o p r o p v l i d e n e-\alpha-D-l y x o f u r a n o s e$, the monoacetone-Dlyxose of Levene and Tipson. R. Schaffer.

Effect of perchloryl fluoride additions of the flame speed of methane. C. Halpern.

Journal of Research 65C (Eng. and Instr.), No. 4 (Oct.-Dec. 1961) 70 cents.

A new airglow photometer. C. M. Purdy, L. R. Megill, and F. E. Roach.

A guide to the use of the modified reflectometer technique of VSWR measurement. W. J. Anson.

Apparatus for determination of pressure-density-temperature relations and specific heats of hydrogen to 350 atmospheres at temperatures above $14^{\circ} \mathrm{K}$. R. D. Goodwin. 
An $\mathrm{X}$-ray diffractometer cryostat providing temperature control in the range 4 to $300^{\circ} \mathrm{K}$. F. A. Mauer and L. H. Bolz.

The use of a thermistor for detecting eluent fronts in liquidsolid chromatography. G. S. Ross.

Radiation field from a circular disk source. J. H. Hubbell, R. L. Bach, and R. J. Herbold.

The Bauschinger effect and residual microstresses in alpha brass. V.J. Newton.

A study by polarization techniques of the corrosion rates of aluminum and steel underground for sixteen months. W. J. Schwerdtfeger.

Journal of Research 65D (Radio Prop.), No. 5 (Sept.-Oct. 1961) 75 cents.

Frequency dependence of $D$-region scattering at VHF. J. C. Blair, R. N. Davis, Jr., and R. C. Kirby.

Theoretical scattering coefficient for near vertical incidence from contour maps. H. S. Hayre and R. K. Moore.

Mutual interference between surface and satellite communication systems. W. J. Hartman and M. T. Decker.

VHF and UHF signal characteristics observed on a long knife-edge diffraction path. A. P. Barsis and R. S. Kirby.

Experimental study of inverted L-, T-, and related transmission-line antennas. S. Prasad and R. W. P. King.

Reflection from a sharply bounded ionosphere for VLF propagation perpendicular to the magnetic meridian. D. D Crombie.

Resonance of the space between earth and ionosphere. H. Poeverlein.

Observed attenuation rate of ELF (region below $1 \mathrm{kc} / \mathrm{s}$ ) radio waves. A. G. Jean, A. C. Murphy, J. R. Wait, and D. F. Wasmundt.

A note concerning the excitation of ELF electromagnetic waves. J. R. Wait.

Computation of whistler ray paths. I. Yabroff.

On the analysis of LF ionospheric radio propagation phenomena. J. R. Johler.

Journal of Research 65D (Radio Prop.), No. 6 (Nov.-Dec. 1961) 75 cents.

The solar wind. E. N. Parker.

Attentuation coefficients for propagation at very low frequencies (VLF) during a sudden ionospheric disturbance (SID). E. T. Pierce.

Dipole radiation in a conducting half space. R. K. Moore and W. E. Blair.

Reliability of atmospheric radio noise predictions. J. R. Herman.

Effects of the ionosphere on VLF navigational aids. W. T. Blackband.

On the spectrum of terrestrial radio noise at extremely low frequencies. H. R. Raemer.

The nonsingular embedding of transition processes within a more general framework of coupled variables. J. Heading. (See above abstract.)

Worldwide VLF standard frequency and time signal broadcasting. A. D. Watt, R. W. Plush, W. W. Brown, and A. H. Morgan.

Design of panoramic ionospheric resources. L. H. Heisler and L. D. Wilson.

A quick method for estimating the stage of the sunspot cycle. W. B. Chadwick.

Measurements of low-angle radiation from a monopole. A C. Wilson.

Ideal gas thermodynamic functions and isotope exchange functions for the diatomic hydrides, deuterides, and tritides, L. Haar, A. S. Friedman, and C. W. Beckett, NBS Mono. 20 (1961) $\$ 2.75$

Causes of variation in chemical analyses and physical tests of portland cement, B. L. Bean and J. R. Dise, NBS Mono. 28 (1961) 25 cents.

Thermal expansion of technical solids at low temperatures. A compilation from the literature, R. J. Corruccini and J. J. Gniewek, NBS Mono. 29 (1961) 20 cents.

Capacities of stacks in sanitary drainage systems for buildings, R. S. Wyly and H. N. Eaton, NBS Mono. 31 (1961), 35 cents.
Publications of the National Bureau of Standards July 1, 1957, to June 30, 1960 (Includes titles of papers published in outside journals 1950 to 1959), B. L. Arnold, NBS Misc. Publ 240 (1961) $\$ 2.25$

Mean electron density variations of the quiet ionosphereMay 3, 1959, J. W. Wright, L. R. Wescott, and D. J. Brown, NBS TN40-3 (PB151399-3) (1961) \$1.50.

Bibliography on ionospheric propagation of radio waves (1923-1960), W. Nupen, NBS TN84 (PB161585) (1961) $\$ 7.00$.

Collisions of liquid drops with liquids, O. G. Engel, NBS TN89 (PB161590) (1961) $\$ 1.00$

Determination of the $\mathrm{K}$ fluorescence yield of argon by proportional-counter spectrometry, C. Godeau, NBS TN91 (PB161592) (1961) 50 cents.

On the climatology of ground-based radio ducts and associated fading regions, E. J. Dutton, NBS TN96 (PB161597) (1961) $\$ 1.75$.

A fixed frequency, $9.1 \mathrm{Gc}$, field intensity recording receiver with extremely narrow bandwidth, R. W. Hubbard and J. V. Cateora, NBS TN107 (PB161608) (1961) 75 cents.

Data reduction instrumentation for radio propagation research, W. E. Johnson, NBS TN111 (PB161612) (1961) $\$ 1.00$.

The nature, cause and effect of the porosity in electrodeposits. VII. A microscopic examination of nickel-chromium coatings after atmospheric corrosion, D. W. Ernst and F. Ogburn, Plating 48, No. 4, 491-497 (May 1961).

Physical quantities proposed for radiation measurements, L. S. Taylor, Proc. IXth Intern. Congress of Radiology, July 23-30, 1959 (München, Germany), 1301-1302 (1960).

Improving rectifier circuits, G. F. Montgomery, Electronics p. 86-87 (Apr. 7, 1961)

Fracture characteristics of notched tensile specimens of titanium and a titanium alloy, G. W. Geil and N. L. Carwile, Materials Research and Standards (Am. Soc. Testing $\mathrm{Ma}$ terials, Philadelphia, Pa.), 1, No. 1, 16-21 (Jan. 1961).

Absence of an isotope effect in the fractional recrystallization of alpha-D-glucose-1-t, H. S. Isbell, H. L. Frush, and N. B. Holt, Anal. Chem. 33, 225-226 (Feb. 1961).

Comparison of national standards for roentgen measurement, H. O. Wyckoff, IXth Intern. Congress of Radiology, July 23-30, 1959 (München, Germany), 1315-1318 (1960).

Environmental factors in a family-size underground shelter, R. P. Achenbach, Proc. Meeting on Environmental Engineering in Protective Shelters, Feb. 8-10, 1960, Natl. Acad. Sci.-Natl. Research Council, Washington, D.C., 69-118 (1961).

How to evaluate accuracy, W. J. Youden, Materials Research and Standards (Am. Soc. Testing Materials, Philadelphia, Pa.) 1, No. 4, 268-271 (April 1961).

Improved standard for the calibration of vibration pickups, R. R. Bouche, Exp. Mech. 1-6 (April 1961).

Absorption and scattering of photons by holmium and erbium, E. G. Fuller and E. Hayward, Proc. Intern. Conf. Nuclear Structure, 760-766 (Kingston, Canada, 1960).

Introductory remarks, U. Fano, Conf. Coherence Properties of Electromagnetic Radiation, AFOSR-583, Report on Conf., 31-38 (University of Rochester, The Institute of Optics, Rochester, N.Y., Apr. 1961).

Available heat sinks for protected underground installations, B. A. Peavy, Proc. Meeting on Environmental Engineering in Protective Shelters, Feb. 8-10, 1960, Natl. Acad. Sci.Natl. Research Council, Washington, D.C., 69-118 (1961).

Interpretation of the appearance potentials of secondary ions, M. B. Wallenstein and M. Krauss, J. Chem. Phys. 34, No. 3, 929-936 (Mar. 1961).

Observations on the chemiluminescent reaction of nitric oxide with atomic oxygen, H. P. Broida, H. I. Schiff, and T. M. Sugden, Trans. Faraday Soc. 5\%, No. 458, 259-265 (Feb. 1961)

Studies of the low-temperature distillation of hydrogen isotopes, T. M. Flynn, Cryogenies 1, No. 2, 1-5 (Dec. 1960); Advances Cryogenic Engr. 6, 236-244 (1961).

Inert enclosed pump for shaped flow of ultraclean solutions, M. W. Barnes and R. H. Noyce, Rev. Sci. Instr. 32, No. 3, 353 (Mar. 1961).

Radiation protection standards, L. S. Taylor, Radiology $\mathbf{7 4 ,}$ 824-831 (May 1960). 
Atomic beam frequency standards, R. C. Mockler, R. E. Beehler, and C. S. Snider, IRE Trans. Instrumentation I-9, No. 2, 120-132 (Sept. 1960).

The development of more stable gage blocks, M. R. Meyerson, T. R. Young, and W. R. Ney, ASTM Bull. 1, No. 5, 368 374 (May 1961).

The power spectrum and its importance in precise frequency measurements, J. A. Barnes and R. C. Mockler, IRE Trans. Instrumentation I-9, No. 2, 149-155 (Sept. 1960).

VLF phase perturbation associated with meteor shower ionization, C. J. Chilton, J. Geophys. Research 66, No. 2, 379-383 (Feb. 1961)

Departures from the Saha equation for ionized helium. I. Condition of detailed balance in the resonance lines, R. N. Thomas and J. B. Zirker, Astrophys. J. 133, No. 2, 588 595 (Mar. 1961).

Tests for regression coefficients when errors are correlated, M. M. Siddiqui, Annals Math. Stat. 31, No. 4, 929-938 (Dec. 1960)

Electron diffraction studies on solid $\alpha$-nitrogen, E. M. Horl and L. Marton, Acta Crystallographica 14, Pt. I, 11-19 (Jan. 10, 1961).

A comment on the NRL solar Lyman-Alpha results, J. T. Jefferies and R. N. Thomas, Astrophys. J. 133, No. 2, 606-607 (Mar. 1961).

The nation's electronic standards program: where do we now stand? H. W. Lance, IRE Trans. Instrumentation I-9, No, 2, 94-100 (Sept. 1960).

Hard gallium alloys for use as low contact resistance electrodes and for bonding thermocouples into samples, G. C. Harman, Rev. Sci. Instr. 31, No. 7, 717-720 (July 1960).

Scattering of photons by deformed nuclei, E. G. Fuller and E. Hayward, Proc. Intern. Conf. Nuclear Structure, 763766 (Kingston, Canada, 1960)

The glass electrode, R. G. Bates, Book, Reference Electrodes, edited by D. J. G. Ives and G. J. Janz (Academic Press Inc. New York, N. Y.), Chapter 5, 231-269 (1961).

A recalibration of the NBS standard thermal neutron flux, E. R. Mosburg, Jr., and W. M. Murphey, J. Nuclear Eng. (Reactor Science and Technology Pt.) 14, 25-30 (1961).

The relationship of low-height ionosonde echoes to Auroralzone absorption and VHF D-scatter, J. K. Olesen and J. W. Wright, J. Geophys. Research 66, No. 4, 1127-1134 (Apr 1961).

Trends in the technology of automatic data processing, S. N. Alexander, A MA Report 43, 38-42 (1960).

Use of the bulge test for determining mechanical properties of stainless steel foil, D. B. Ballard, Materials Research and Standards 1, No, 6, 471-473 (June 1961).

Some comments on the use and preparation of hard gallium alloys, G. C. Harman, Rev. Sei. Instr. 32, No. 4, 465-466 (Apr.1961)

On the nature of equatorial spread, $F$, R. Cohen and K. L. Bowles, J. Geophys. Research 66, No. 4, 1081-1106 (Apr. 1961).

A study of the chemical reactions in kilning bone char, F. G. Carpenter, and V. R. Deitz, Proc. 6th Tech. Session on Bone Char 1959, p. 321 (Bone Char Research Project Inc., Charlestown, Mass., 1961).

Rate of vaporization of refractory substances, R. F. Walker, J. Efimenkof, and N. L. Lofgren, Book, Physical Chemistry in Aerodynamics and Space Flight (Pergamon Press, Inc., Lond on, England), p. 24-30 (1961).

Analysis of pyrolyzates of polystyrene and Poly(methyl methacrylate) by gas chromatography, F. A. Lehmann and G. M. Brauer, Anal. Chem. 33, No. 6, 673-676 (May 1961).

A note on phase velocity of VLF radio waves, J. R. Wait and K. P. Spies, J. Geophys. Research 66, No. 3, 992-993 (Mar. 1961)

Microwave spectrum of isobutylene, Dipole moment, internal barrier, equilibrium conformation, and structure, V. W. Laurie, J. Chem. Phys. 34, No. 5, 1516-1519 (May 1961).

Intermolecular forces from optical spectra of impurities in molecular crystals, R. W. Zwanzig, Molecular Phys. 3, No. 4, 305-311 (July 1960).

On the excitation of electromagnetic surface waves on a curved surface, J. R. Wait, IRE Trans. Ant. Prop. AP-8, No. 4, 445-448 (July 1960).
Solubility product phenomena in hydroxyapatite-water systems, H. M. Rootare, V. R. Deitz and F. G. Carpenter, Proc. 6th Tech. Session on Bone Char 1959, p. 287 (Bone Char Research Project Inc., Charlestown, Mass., 1961).

Reproducibilities of carbon and germanium thermometers at $4.2^{\circ} \mathrm{K}, \mathrm{M}$. H. Edlow and H. H. Plumb, Proc. Cryogenic Eng. Conf., Boulder, Colo., Aug. 1960, 6, 542-547 (1961).

The automatic chloride titrator in the analysis of some ionic constituents, M. D. Peiperl, F. G. Carpenter, and V. R. Deitz, Proc. 6th Tech. Session on Bone Char 1959, p. 53 (Bone Char Research Project Inc., Charlestown, Mass, 1961)

Spin-lattice relaxation in cerous magnesium nitrate, $R$. $P$ Hudson and R. S. Kaeser, Il Nuovo Cimento 19, Serie X, 1275-1277 (Mar. 16, 1961).

The photolysis of ethyl vinyl ether. E. Murad, J. Am. Chem. Soc. 83, 1327-1330 (1961).

Vapor phase gamma-radiolysis of azomethane, L. J. Stief and P. Ausloos, J. Phys. Chem, 65, 877-881 (1961).

Electron interferometer studies of iron whiskers, H. A. Fowler, L. Marton, J. A. Simpson, and J. A. Suddeth, J. Appl. Phys. 32, No. 6, 1153 (June 1961).

Changes in dentures during storage in water and in service, J. B. Woefel, G. C. Paffenbarger, and W. T. Sweeney, J. Am. Dental Assoc. 62, No. 6, 643-657 (June 1961).

Solar disturbances and radio communication forecasts, J. F. Brockman, Sky and Telescope XXI, No. 6, 322-326 (June 1961)

Dissociation constant of the protonated acid form of 2-Amino2-(hydroxymethyl)-1, 3-propanediol [tris(hydroxymethyl) aminomethane] and related thermodynamic quantities from 0 to $50^{\circ}$, R. G. Bates and H. B. Hetzer, J. Phys. Chem. 65, $667-671$ (1961).

Hyperfine structure and isotope shifts in the 2537-A line of mercury, W. G. Schweitzer, Jr., J. Opt. Soc. Am. 51, No. 6 , 692-693' (June 1961).

Long-distance one-hop $F_{1}$ propagation through the auroral zone, L. H. Tveten, J. Geophys. Research 66, No. 6 , 1683-1684 (June 1961).

Resonators for millimeter and submillimeter wavelengths, W. Culshaw, IRE Trans. Microwave Theory and Tech., MTT-9, No. 2, 135-144 (March 1961).

Paramagnetic substances for nuclear orientation, R. P. Hudson, Prog. in Cryogenics, 3, 99-127 (1961).

A five-attribute system of describing visual appearance, D. B. Judd, Am. Soc. Testing Materials Spec. Tech. Pub. No. 297 (1961).

Circumferentially uniform electroplating of tube bores, J. P. Young and H. I. Salmon, Plating 48, No. 7, 783; 787-8 (July 1961)

Solar flare effects in the $F$ region of the ionosphere, R. W Knecht and K. Davies, Nature 190, No. 4778, 797-798 (May 1961).

Age to indium resonance for D-D neutrons in heavy water, V. Spiegel, Jr., and A. C. B. Richardson, Nuclear Sci. and Eng. 10, No. 1, 11-15 (1961).

Magnetic properties and optical absorption spectrum of $\mathrm{K}_{2} \mathrm{ReCl}_{6}$, J. C. Eisenstein, J. Chem. Phys. 34, No. 5, 1628 1648 (May 1961)

Convective circulation in water induced by evaporation cooling, W. G. Spangenberg and W. R. Rowland, Phys. of Fluids, 4, No. 6, 743-750 (June 1961).

Photoionization heating in the $F$ region of the atmosphere, D. C. Hunt and T. E. Van Zandt, J. Geophys. Research 66, No. 6, 1673-1682 (June 1961).

Identification of fiber blends by infrared spectroscopy, M. K. Wharton and F. H. Forziati, Am. Dyestuff Rep. 50, No. 14, 33-36 (July 1961).

Residual losses in a guard-ring micrometer-electrode holder for solid-disk dielectric specimens, A. H. Scott and W. P. Harris, 1960 Annual Report, Conference on Electrical Insulation, Natl. Acad. Sci.-Natl. Research Council, 11-17 (1961).

Calculations of the neutron age in water and heavy water for D-D sources, J. W. Cooper, Nuclear Sci. and Eng. 10, No. 1, 1-10 (May 1961)

Magnetic susceptibility of cerous magnesium nitrate, R. P. Hudson and W. R. Hosler, Phys. Rev. 12\%, No. 5, $1417-$ 1420 (June 1961). 
Use of gaussian orbitals for atoms-in-molecule calculations, M. Kraus, J. Chem. Phys. 34, No. 2, 692-693 (February 1961).

Measurement of minority carrier lifetime in SiC by a novel electroluminescent method, G. C. Harman and R. L. Raybold, J. Appl. Phys. 32, No. 6, 1168-1169 (June 1961).

Absolute measurement of $\mathrm{W}$ for $\mathrm{Po}^{210}$ alpha particles in air, nitrogen, and carbon dioxide, Z. Bay, P. A. Newman, and H. H. Seliger, Radiation Research 14, No. 5, 551-565 (May 1961)

Comparative $p \mathrm{H}$ measurements on papers by water extraction and glass electrode spot tests, J. H. Flynn and L. E. Smith, Tappi 44, No. 3, 223-228 (Mar. 1961).

The structure of the monoclinic form of sodium tetrametaphosphate tetrahydrate, H. M. Ondik, C. H. MacGillavry, and S. Block, Acta. Crystallographica 14, 555 (1961).

A comparison between theoretical and experimental data on phase velocity of VLF radio waves, J. R. Wait, Proc, IRE 49, No. 6, 1089-1090 (June 1961).

Mass spectrometric study of cyanogen and cyanoacetylenes, V. H. Dibeler, R. M. Reese, and J. L. Franklin, J. Am. Chem. Soc. 83, 1813-1818 (1961).

An analysis of errors in the calibration of electric instruments, F. L. Hermach, Communication and Electronics, 1-6 (May 1961).

Energy levels and magnetic dipole transitions in the $4 p^{4}$ ground configuration of singly ionized atomic bromine (Br II), W. C. Martin and J. L. Tech, J. Opt. Soc. Am. 51, No. 6, 591-594 (June 1961).

An improved structure determination for vinyl fluoride, D. R. Lide, Jr., and D. Christensen, Spectrochim. Acta 17, 665668 (1961).

Incoherent scattering by free electrons as a technique for studying the ionosphere and exosphere: Some observations and theoretical considerations, K. L. Bowles, pp. 223-243 of AGARDograph 42, The upper atmosphere above F2maximum. North Atlantic Treaty Organization, Advisory Group for Aeronautical Research and Development, Avionics Panel. Papers presented at the Symposium of the Ionospheric Research Committee, Paris, France, May 1959.

A $p \mathrm{H}$ standard for blood and other physiologic media, V. E. Bower, M. Paabo, and R. G. Bates, Clin. Chem. 7, No. 3, 292 (June 1961).
Evidence on the laminar nature of the exosphere obtained by means of guided high-frequency wave propagation, R. M. Galley and W. F. Utlaut, Phys. Rev. Letters 6, No. 11, 591-595 (June 1, 1961).

Comparison of the ionization produced in air by alpha particles near 5 mev and by beta particles, Z, Bay and P. A. Newman, Radiation Research 14, No. 5, 566-572 (May 1961).

A century-old razor, S. J. Rosenberg, Metal Progress, 94-96 (1961).

Some applications of Hardy's formula in Brownian motion (Abstract), A. Ghaffari, Am. Math. Soc. Notices 8, No. 54, 238 (June 1961).

On the classical approximation in the statistical theory of mass spectra, H. M. Rosenstock, J. Chem. Phys. 34, 2182 (1961).

Optical calibration of vibration pickups at small amplitudes, V. A. Schmidt, S. Edelman, E. R. Smith, and E. Jones, J. Acoust. Soc. Am. 33, No. 6, 748-751 (June 1961).

Superconductivity of $\mathrm{Nb}_{3} \mathrm{Sn}$ in pulsed fields of 185 kilogauss, V. D. Arp, R. H. Kropschot, and J. H. Wilson, Phys. Rev. Letters 6, No. 9, 452-453 (May 1961).

Proposed nomenclature for linear viscoelastic behavior, $H$. Leaderman and F. Schwarzl, Rheologica Acta, 1, No. 4/6, 674-676 (1961).

FM and SSB radiotelephone tests on a VHF ionospheric scatter link during multipath conditions, IRE Trans. Commun. Systems CS-8, No. 3, 813-186 (Sept. 1960).

* Publications for which a price is indicated (except for Technical Notes) are available only from the Superintendent of Documents, U.S. Government Printing Qffice, Washington 25, D.C. (foreign postage, one-fourth additional). Technical Notes are available only from the Office of Technical Services, U.S. Department of Commerce, Washington 25, D.C. (order by PB number). The Technical News Bulletin and Basic Radio Propagation Predictions are available on a 1-, 2-, or 3 -year subscription basis, although no reduction in rates can be made. Reprints from outside journals and the NBS Journal of Research may often be obtained directly from the authors. 